\title{
Tetanus in Deutschland - Ergebnisse der Einzelfallerfassung seit 1995
}

\section{Einführung}

Anknüpfend an einen früher publizierten Bericht zum Auftreten von Erkrankungen und Sterbefällen an Tetanus in Deutschland [1] soll dargestellt werden, wieviel Tetanus-Erkrankungen in den letzten Jahren gemeldet worden sind, wodurch sie bedingt waren und welche Bedeutung den Tetanus-Erkrankungen zum gegenwärtigen Zeitpunkt zukommt. Grundlage hierfür sollen einerseits die Zahlen der Erkrankungen sowie der Sterbefälle, herausgegeben vom Statistischen Bundesamt, sein $[2,3]$. Andererseits sollen in einer ersten Auswertung die vom Robert Koch-Institut (RKI) im Rahmen von Sondererhebungen seit 1995 erhaltenen Angaben zu den einzelnen Fällen zusammengefaßt werden.

\section{Sondererhebungen von Erkrankungen an Tetanus}

Tetanus gehört zu den nach $\$ 3$ BSeuchG meldepflichtigen Erkrankungen. Nach Eingang der wöchentlich aus den Regierungsbezirken und Bundesländern an das RKI gegebenen Meldungen wird ein spezieller Tetanus-Erhebungsbogen an die entsprechenden $\mathrm{Ge}$ sundheitsämter übersandt, mit der Bitte, anhand des Bogens detaillierte Informationen zum einzelnen Erkrankungsfall zu übermitteln. Die Weitergabe der $\mathrm{Da}$ ten an das RKI erfolgt streng anonymisiert. Der Erhebungsbogen enthält Angaben zu vorangegangenen Verletzungen bzw. zu bekannten Vorschäden, zum Arztbesuch einschließlich vorgenommener Maßnahmen zur TetanusProphylaxe, zum Verlauf der Erkrankung, aber auch zur Impfanamnese. Die Mitarbeit der Gesundheitsämter erfolgt auf freiwilliger Basis. An dieser Stelle sei allen Kolleginnen und Kollegen sehr herzlich gedankt, die die Sondererhebungen des RKI durch die Übermittlung der Angaben zu den aufgetretenen Erkrankungsfällen an Tetanus unterstützten.

\section{Ergebnisse}

Entsprechend den Veröffentlichungen des Statistischen Bundesamtes wurden
Tabelle 1: Tetanus in Deutschland - Erkrankungen und Sterbefälle [2, 3]

\begin{tabular}{lcc}
\hline Jahr & Erkrankungen & Sterbefälle \\
\hline 1991 & 16 & 2 \\
1992 & 14 & 4 \\
1993 & 16 & 4 \\
1994 & 14 & 6 \\
1995 & 11 & 2 \\
1996 & 17 & liegt noch nicht vor \\
\hline
\end{tabular}

in den zurückliegenden Jahren (seit 1991) die in Tabelle 1 dargestellten Zahlen für Erkrankungen und Sterbefälle an Tetanus in Deutschland registriert. Die Zahl der jährlich gemeldeten Erkrankungen ist etwa gleich groß. Auch Sterbefälle treten in jedem Jahr auf. Nach wie vor haben Erkrankungen an Tetanus in Deutschland eine hohe Letalität. Für den Zeitraum von 1991 bis 1995 errechnet sich auf der Grundlage der dargestellten Angaben eine Letalität von $25 \%$.

Im Rahmen der Sondererhebungen liegen Angaben zu insgesamt 26 Erkrankungsfällen vor. Im einzelnen sind das für das Jahr 1995 insgesamt zehn ausgefüllte Erhebungsbögen (laut Statistischem Bundesamt elf Erkrankungen), für 199614 zurückgesandte Bögen (Zahl der Erkrankungen 17) und zwei Bögen im ersten Halbjahr 1997 (insgesamt zwei Erkrankungen). Die Angaben zu den einzelnen Fällen varïeren hinsichtlich ihrer Vollständigkeit. Sie lassen jedoch zusammenfassende Verallgemeinerungen zu.

Die im beschriebenen Zeitraum aufgetretenen 26 Erkrankungsfälle an Tetanus verteilen sich auf insgesamt 14 Bundesländer. Männer und Frauen waren gleichermaßen von Erkrankungen an Tetanus betroffen (zwölf Personen männlichen Geschlechts, 14 weiblichen Geschlechts). Für 21 der an Tetanus Erkrankten ist bekannt, daß sie deutscher Nationalität sind. Für die fünf übrigen Personen wurde die Nationalität nicht angegeben. Die Tetanuserkrankungen traten vorwiegend bei Personen höheren Lebensalters auf. 13 der 26 Parienten waren älter als 65 Jahre, sieben zwischen 55 und 64 Jahre (Abb. 1). Die jüngste in diesem Zeitraum an Tetanus erkrankte Person war 30 Jahre alt, die älteste 89.

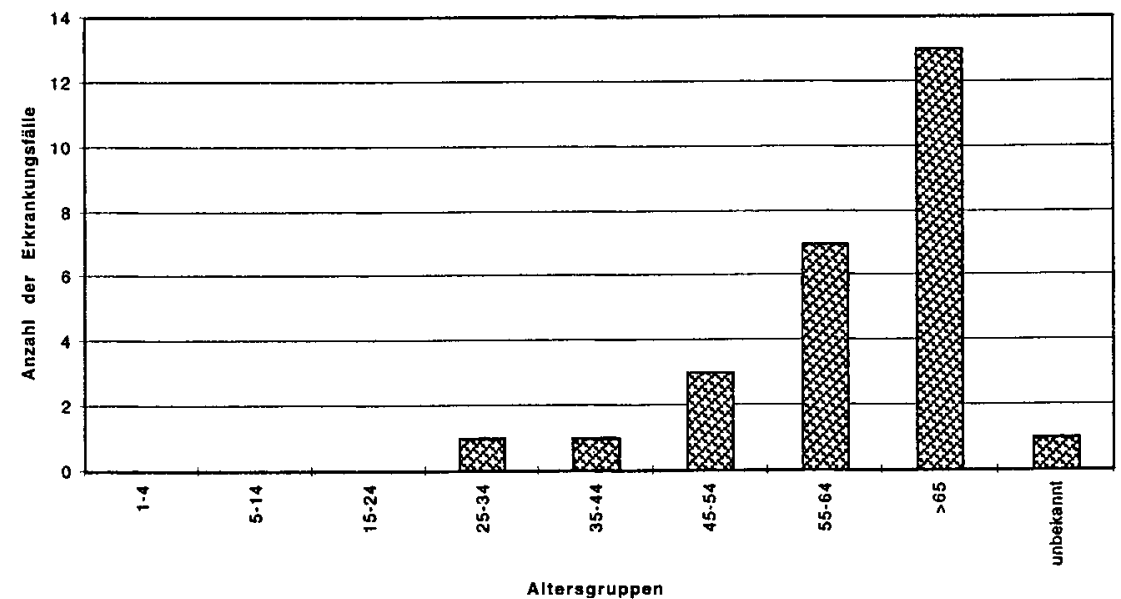

Abbildung 1: Tetanuserkrankungen in Deutschland seit 1995 - Altersgruppen 
Für den allergrößten Teil der Erkrankungsfälle (23 Fälle) sind in der Anamnese Verletzungen bzw. Erfrierungen bekannt. Bei den Verletzungen, die den Tetanuserkrankungen zugrunde lagen, handelte es sich um Riß-, Schürf- und Kratzwunden, die bei der Gartenarbeit, beispielsweise durch Rosen oder Brombeeren, bzw. bei Unfällen (nach Sturz oder Fahrradunfall) erworben wurden, aber auch um Kreissägeverletzungen oder Verletzungen durch ein Beil. In drei Fällen waren rostige Gegenstände beteiligt. In einem Fall waren Erfrierungen am Fuß vorhanden.

Für eine der erfaßten Erkrankungen ohne vorangegangene Verletzung - war ein Ulcus cruris in der Anamnese bekannt. Für eine weitere Erkrankung, be der keine Verletzung vorlag, wurde erhoben, daß eine laparoskopische Cholecystektomie neun Tage vor Erkrankungsbeginn bei der betreffenden Patientin vorgenommen worden war. Für einen Erkrankungsfall liegt keine Angabe vor.

Der Zeitraum von der Verletzung bis zum Auftreten der Tetanus-Symptomatik lag zwischen drei Tagen und drei Wochen (Angaben für 17 Fälle). Im größten Teil der Fälle kam es bereits in den ersten zehn Tagen zur Erkrankung (14 Fälle). Dies entspricht den in der Literatur angegebenen Zeiträumen. Der Erkrankungsfall mit der längsten Inkubationszeit (ca. drei Wochen nach Verletzung) trat bei einem Floristen auf, nachdem sich dieser an einer Rose gestochen hatte.

Hinsichtlich des Impfstatus der Erkrankten liegen Angaben für 15 der 26 Personen vor. Vier Patienten erkrankten trotz früher durchgeführter Grundimmunisierung, wobei die Zeitabstände von der zuletzt durchgeführten Impfung bis zur Erkrankung nicht in allen Fällen bekannt waren. Drei Erkrankte waren unvollständig geimpft worden. Acht der Erkrankten waren - den Angaben zufolge - vorher nicht geimpft worden.

Nur ein Teil der an Tetanus Erkrankten suchte unmittelbar nach der Verletzung einen Arzt auf (bei sechs der 26 Erkrankten bekannt). Elf Patienten nahmen ärztliche Hilfe erst in Anspruch, als Erkrankungssymptome schon aufgetreten waren. Für zwei Patienten ist ein Arztbesuch in der Inkubationszeit, drei bzw. vier Tage vor Auftreten der Symptome, bekannt. (Für acht Erkrankungsfälle liegen hierzu keine Angaben vor.) Bei den Patienten, die vor Auftreten der Symptome einen Arzt aufgesucht hatten, wurden in vier Fällen keine Maßnahmen der Tetanus-Prophylaxe eingeleitet. In drei Fällen wurde Tetanus-Impfstoff verabreicht, einmal Immunglobulin.

Bezogen auf alle in die Auswertungen aufgenommenen Tetanuserkrankungen wurden folgende Prophylaxe-Maßnahmen vorgenommen: in elf Fällen Impfstoff plus Immunglobulin, in vier Fällen nur Impfstoff, zweimal nur Immunglobulin. Bei sechs Patienten wurden überhaupt keine prophylaktischen Maßnahmen eingeleitet, für drei Fälle liegen hierzu keine Angaben vor.

Die Diagnostik der Tetanuserkrankung beruhte in fast allen Fällen auf dem Vorliegen klinischer Symptome (für eine Erkrankung ohne Angabe). In einem Fall erfolgte zusätzlich ein Erregernachweis.

Im Rahmen der Sondererhebungen liegen Angaben zu fünf Sterbefällen infolge Tetanus vor. Vier der Verstorbenen waren zwischen 76 und 86 Jahre alt (bei einem Fall keine Altersangabe). Für alle fünf Fälle ist unbekannt, ob frühere Impfungen vorgenommen worden waren oder nicht.

\section{Diskussion}

Tetanuserkrankungen spielen nach wie vor auch in Deutschland eine Rolle, insbesondere wegen des oftmals tödlichen Verlaufs. Betrachtet man die Zahl der auftretenden Erkrankungen, so wird deutlich, daß diese seit dem Ende der achtziger Jahre unverändert ist. Ein Vergleich mit den Erkrankungszahlen in anderen Ländern zeigt, daß in den USA etwa gleich große Inzidenzraten wie in Deutschland erreicht werden $(0,02 \mathrm{Er}$ krankungen pro 100000 Einwohner) [4]. Auch für Großbritannien werden nur einige Erkrankungsfälle pro Jahr ausgewiesen (Tab. 2). In einigen eu-

Tabelle 2: Erkrankungen an Tetanus in ausgewählten Ländern $[4,5]$

\begin{tabular}{lccc}
\hline Land/Jahr & 1992 & 1993 & 1994 \\
\hline USA & 45 & 48 & 51 \\
Großbritannien & 8 & 8 & 4 \\
Frankreich & 54 & 52 & 35 \\
Italien & 92 & 90 & 102 \\
Spanien & 51 & 48 & 36 \\
Türkei & 110 & 95 & 105 \\
\hline
\end{tabular}

ropäischen Ländern werden - den Angaben der WHO entsprechend - hingegen höhere Erkrankungszahlen an Tetanus registriert als in Deutschland. Dazu gehören Frankreich, Italien, Spanien und die Türkei [5].

Auch wenn die Zahl der Sterbefälle durch Tetanus in Deutschland im Vergleich zu den achtziger Jahren leicht rückläufig ist, so ist doch die Letalität von $25 \%$ noch immer recht hoch. Ein Vergleich mit den beispielsweise in den USA beobachteten Tetanusfällen zeigt jedoch, daß auch dort eine Letalität von $25 \%$ ermittelt wurde [4].

Aus den Ergebnissen der Sondererhebungen geht hervor, daß nur ein Teil der an Tetanus Erkrankten rechtzeitig, d. h. unmittelbar nach Verletzung, einen Arzt aufsuchte. Nicht in allen Fällen wurden durch den Arzt die bestmöglichen prophylaktischen Maßnahmen eingeleitet, wie sie den 1997 publizierten STIKO-Empfehlungen zu entnehmen sind [6].

Im Ergebnis der Erhebungen wurde deutlich, daß die Impfanamnese der an Tetanus erkrankten Patienten sehr verschieden war. Ein großer Teil der Betroffenen war früher unzureichend oder gar nicht geimpft worden. Auch bei vorhandener Grundimmunisierung wurden nicht in allen Fällen Boosterungen im Abstand von zehn Jahren vorgenommen. Andere Patienten weisen dagegen vollständig durchgeführte Impfungen entsprechend den Empfehlungen auf.

Auswertungen aller von 1991 bis 1994 in den USA registrierten Tetanusfälle ergaben ein ähnliches Bild [4]. Ca. $25 \%$ der Erkrankten waren ungeimpft, während etwa $12 \%$ unzureichend geimpft waren. Über $9 \%$ der Erkrankten hatten jedoch mehr als vier Impfstoff-Dosen erhalten. Dabei ist für zwei dieser Patienten bekannt, daß sie vier bzw. sechs Jahre vor der Erkrankung die letzte Boosterung bekommen hatten. 
Für rund die Hälfte aller Tetanuserkrankten (54 \%) blieb die Impfanamnese unbekannt.

Tetanus ist eine Erkrankung hauptsächlich des höheren Lebensalters. Erwartungsgemäß waren auch bei den Erhebungen des RKI in den Jahren 1995 bis 1997 vorwiegend ältere Jahrgänge vertreten. Lediglich zwei der an Tetanus Erkrankten waren bis zu 40 Jahre alt (30 bzw. 40 Jahre).

Eine in den USA durchgeführte Studie, an der mehr als 10000 Personen verschiedener Altersgruppen beteiligt waren, zeigte, daß die Antikörper-Titer gegen Tetanus mit zunehmendem Alter abnehmen. Bei den über 70jährigen war eine Tetanusimmunität nur noch bei $28 \%$ der Probanden nachweisbar [7].

Hinweise auf eine unzureichende Tetanusimmunität - insbesondere bei Personen höheren Lebensalters - geben auch die in Deutschland vorgenommenen Untersuchungen von Thilo (1994), in die mehr als 4000 Personen verschiedener Altersgruppen einbezogen worden waren [8]. Sind 30- bis 40jährige Männer noch zum allergrößten Teil gegen Tetanus geschützt (nur 3,6\% ungeschützte in den alten Bundesländern), so ergibt sich bei den 60 - bis 70jährigen ein ganz anderes Bild. 68,6 \% der Frauen und 44,6 \% der Männer dieser Altersgruppe in den alten Bundesländern sind gegen Tetanus ungeschützt. In den neuen Bundesländern trifft dies für $11,9 \%$ der 60 bis 70 jährigen Frauen und für $7,7 \%$ der Männer zu.

$\mathrm{Zu}$ ähnlichen Ergebnissen kamen Steger und Mitarbeiter (1996), die in Österreich Personen < 30 Jahre $(\mathrm{n}=25)$ und Personen $>65$ Jahre $(n=32)$ hinsichtlich ihrer Tetanus-Immunität untersuchten. Die Autoren stellten fest, daß $60 \%$ der älteren Personen ungeschützt gegen Tetanus sind. Waren zwei Drittel dieser Personen gar nicht oder unvollständig geimpft gewesen, so war ein weiteres Drittel der als "ungeschützt « eingestuften Personen entsprechend den
Empfehlungen geimpft worden (Booster-Impfungen alle $10 \mathrm{Jahre)}$ [9].

Offen bleibt zum gegenwärtigen Zeitpunkt, ob der Nachweis oder das Fehlen von spezifischen Antikörpern generelle Rückschlüsse auf die tatsächliche $\mathrm{Ge}$ fährdung der Personen zuläßt oder nicht.

Nach Baily [10] ist es nicht gerechtfertigt, den fehlenden Nachweis von spezifischen Antikörpern mit einem ungenügenden Schutz gleichzusetzen. Zum einen bedeutet die verhältnismäßig große Zahl der Personen ohne nachweisbare Tetanus-spezifische Antikörper nicht notwendigerweise auch eine große Zahl von Tetanuserkrankungen in der Bevölkerung, wie sie vor Einführung der Tetanusimpfung registriert werden mußte. Andererseits wurden in der Literatur auch Fälle beschrieben, wo es zu Tetanuserkrankungen kam, obwohl hohe Antikörper-Titer nachgewiesen werden konnten.

Generell kann jedoch festgestellt werden, daß Tetanus in Ländern, die gut etablierte Impfprogramme haben, sehr selten ist. Die Tatsache, daß für Ältere das Risiko, an Tetanus zu erkranken, am höchsten ist, spiegelt meist eine unzureichende Immunisierung wider, oft ohne abgeschlossene Grundimmunisierung.

So sollten die auch heute noch in Deutschland auftretenden Erkrankungsfälle an Tetanus Anlaß sein, dem Impfschutz aller Bürger, dem der jüngeren wie der älteren, besondere Aufmerksamkeit zu schenken. Das trifft für Tetanus zu, aber genauso auch für weitere Infektionskrankheiten, wie z. B. die Diphtherie.

Jeder sollte entsprechend den aktuellen Impfempfehlungen der STIKO [6] auch gegen Tetanus geimpft werden, um im Verletzungsfall möglichst geschützt zu sein.

Die STIKO-Empfehlungen machen deutlich, daß ein optimaler Impfschutz angenommen werden kann, wenn bei vorhandener Grundimmunisierung alle zehn Jahre eine Auffrischimpfung verabreicht wurde. Im Verletzungsfall ist, in Abhängigkeit von Art und Ausmaß der Verletzung sowie dem Vorliegen früherer Impfungen, zu entscheiden, welche der Prophylaxe-Maßnahmen vorgenommen werden sollten, oder obbei vollendeter Grundimmunisierung und erst kürzlich erfolgter BoosterImpfung sowie einer geringfügigen, sauberen Wunde - keine dieser Maßnahmen notwendig ist.

Literatur:

[1] Zastrow, K.-D., und Schöneberg, I.: Tetanus - Erkrankungen, Impfungen und Impfschäden in der Bundesrepublik Deutschland 1971 bis 1990. Dtsch. med. Wschr. 118 (1993), 1617-1620.

[2] Statistisches Bundesamt: Gesundheitswesen, Fachserie 12, Reihe 2: Meldepflichtige Krankheiten. Wiesbaden (jährlich).

[3] Statistisches Bundesamt: Gesundheitswesen, Fachserie 12, Reihe 4: Todesursachen. Wiesbaden (jährlich).

[4] Izurieta, H. S., Sutter, R. W., Strebel, P. M., Bardenheier, B., Prevots, D. R., Wharton, M., and Hadler, S. C.: Tetanus surveillance United States, 1991-1994. MMWR, 46 (1997) SS-2.

[5] WHO: Expanded Programme on Immunization. Information System. Summary for the WHO European Region (1995).

[6] Impfempfehlungen der Ständigen Impfkommission (STIKO). Stand: März 1997. Epidemiologisches Bulletin 15/97 97-108.

[7] Gergen, P. J., McQuillan, G. M., Kiely, M., Ezzati-Rice, T. M., Sutter, R. W., and Virella, G.: A population-based serologic survey of immunity to tetanus in the United States. New Engl J Med 332 (1995) 761-766.

[8] Thilo, W.: Die novellierten STIKO-Empfehlungen. Der Allgemeinarzt 16 (1994) 5, 380-384.

[9] Steger, M. M., Maczek, C., Berger, P., and Grubeck-Loebenstein, B.: Vaccination against tetanus in the elderly: do recommended vaccination strategies give sufficient protection? Lancet 348 (1996) 762

[10] Baily, G.: Are the elderly inadequately protected against tetanus? Lancet 348 (1996) 1389-1390.

Anschrift der Verfasser:

Dr. Gernot Rasch, Dr. Irene Schöneberg, Robert Koch-Institut, Fachgebiet 411, Stresemannstr. 90-102, 10963 Berlin 